\title{
Hepatitis B immunoglobulin prophylaxis for de novo hepatitis B infection in liver transplantation: a 30-year experience
}

\author{
Hye-Sol Jung ${ }^{1}$, YoungRok Choi ${ }^{1}$, Kyung Chul Yoon ${ }^{2}$, Su Young Hong ${ }^{1}$, Sanggyun Suh ${ }^{1}$, Kwangpyo Hong ${ }^{1}$, \\ Eui Soo Han ${ }^{1}$, Jeong-Moo Lee ${ }^{1}$, Suk Kyun Hong ${ }^{1}$, Nam-Joon Yi ${ }^{1}$, Kwang-Woong Lee ${ }^{1}$, Kyung-Suk Suh ${ }^{1}$ \\ ${ }^{1}$ Department of Surgery, Seoul National University College of Medicine, Seoul National University Hospital, Seoul, Korea; ${ }^{2}$ Department of Surgery, \\ Seoul National University College of Medicine, Seoul National University Boramae Medical Center, Seoul, Korea \\ Contributions: (I) Conception and design: Y Choi, NJ Yi, KW Lee, KS Suh; (II) Administrative support: SY Hong, S Suh, K Hong, ES Han, JM Lee; \\ (III) Provision of study materials or patients: Y Choi, KC Yoon; (IV) Collection and assembly of data: SY Hong, S Suh, K Hong, ES Han, JM Lee; (V) \\ Data analysis and interpretation: HS Jung, Y Choi; (VI) Manuscript writing: All authors; (VII) Final approval of manuscript: All authors. \\ Correspondence to: YoungRok Choi. Department of Surgery, Seoul National University College of Medicine, Seoul National University Hospital, 101 \\ Daehak-ro, Jongno-gu, Seoul 03080, Korea. Email: choiyoungrok@gmail.com.
}

Background: Donors positive for hepatitis B core antibody (HBcAb) are an important source of organs in hepatitis B virus (HBV) endemic areas despite the risk of occult infection. We analyzed the long-term outcomes of hepatitis B immunoglobulin in de novo HBV prevention following liver transplantation (LT) using HBcAb-positive grafts.

Methods: The prospectively collected data from 2,201 recipients at Seoul National University Hospital (SNUH) and Seoul National University Boramae Medical Center between 1988 and 2018 were retrospectively reviewed. A total of 1,458 patients were enrolled. Of the 1,458, 478 (32.8\%) grafts were corepositive, 152 (10.4\%) of which belonged to HBV surface antigen-negative recipients. During the anhepatic phase, hepatitis B immunoglobulin 4,000 IU was administered intravenously and daily until postoperative day 3 .

Results: The 152 patients with hepatitis B surface antigen-negative received HBcAb-positive graft. De novo HBV developed in 21 (13.8\%) of these recipients. De novo HBV occurred in 1, 11, 0, and 9 of the $4 \mathrm{HBcAb}$ - and hepatitis b surface antibody (anti-HB)-negative, $49 \mathrm{HBcAb}$-negative and anti-HB-positive, $1 \mathrm{HBcAb}$-positive and anti-HB-negative, and $98 \mathrm{HBcAb}$ - and anti-HB-positive recipients, respectively. Patients with higher Model for End-stage Liver Disease (MELD) score (23.8 \pm 8.7 vs. 19.5 \pm 9.2 ) or HBcAbnegative recipients $(22.6 \%$ vs. $9.1 \%$ ) had a higher risk of de novo infection. The median follow-up and serum HBV surface antigen-positivity detection time was 69 and 18 months, respectively. The median HBV surface antibody titer was $65.0 \mathrm{IU} / \mathrm{L}$ at de novo infection. Nineteen patients of 21 were treated with nucleoside analogs (NAs), and seven of 19 achieved seroconversion. No patient died of de novo HBV infection.

Conclusions: With close monitoring of viral serum markers and appropriate initiation of NAs, de novo HBV infection can be prevented and treated appropriately with the hepatitis B immunoglobulin monoprophylaxis protocol.

Keywords: Hepatitis B virus (HBV); de novo infection; prophylaxis; long-term; outcome

Submitted Aug 16, 2021. Accepted for publication Dec 09, 2021.

doi: $10.21037 /$ atm-21-4311

View this article at: https://dx.doi.org/10.21037/atm-21-4311 


\section{Introduction}

In Western countries with a low prevalence of hepatitis $B$ virus (HBV) infection, liver grafts positive for hepatitis $B$ core antibody $(\mathrm{HBcAb})$ have been recognized as marginal. However, core-positive donors are an important organ source in HBV endemic areas despite the risk of occult HBV infection (1-3). Recently, transplantations with $\mathrm{HBcAb}$-positive livers have increased as studies revealed favorable outcomes of de novo $\mathrm{HBV}$ infection in those areas (4-6). The growing organ shortage and acceptable outcomes in transplantations involving livers from HBcAb-positive donors have encouraged the use of these extended grafts in clinical practice (7).

Hepatitis B immunoglobulin (HBIG) and antiviral agents have been used to prevent de novo HBV infection following liver transplantation (LT) at various transplant centers. Several studies have demonstrated de novo HBV infection risk with lamivudine monoprophylaxis $(8,9)$. However, some authors reported that de novo HBV prophylaxis with HBIG and lamivudine was effective for preventing de novo infection (10). A recent study in South Korea reported that the overall incidence of de novo HBV infection was $12.5 \%$ without anti-HBV prophylaxis and recorded no difference in survival between the $\mathrm{HBcAb}$-positive and -negative groups (11). Lee and Takemura et al. reported that 10,000 IU of HBIG monoprophylaxis prevented de novo infection in all 18 and 17 patients, respectively, who were hepatitis B surface antigen (HBsAg)-negative after receiving corepositive livers $(12,13)$.

However, the American Association for the Study of Liver Diseases and European Association for the Study of the Liver have recommended de novo HBV prophylaxis with nucleoside analog (NA) monotherapy to be adequate for a low rate of de novo HBV infection, given the high cost of HBIG and need for intravenous route of administration (14).

The NA prophylaxis regimen is simple and most effective; however, to maintain the HBsAb titer, HBIG and HBV vaccination are still used in South Korea because of the low cost of medical insurance and ease of use of HBIG. This means that long-term data on low-dose HBIG-only prophylaxis is inadequate. We have administered HBIGonly prophylaxis for decades in recipients who received a core-positive graft.

In this study, in a large number of recipients of corepositive livers who received HBIG vaccination for de novo HBV prophylaxis, we analyzed the long-term outcomes, risk factors of de novo infection, and clinical course of patients with de novo $\mathrm{HBV}$ infection.

We present the following article in accordance with the STROBE reporting checklist (available at https://atm. amegroups.com/article/view/10.21037/atm-21-4311/rc).

\section{Methods}

\section{Patient selection}

This retrospective study analyzed prospectively collected data of 2,201 patients who underwent LT between January 1988 and December 2018 at the Seoul National University Hospital (SNUH) and Seoul National University Boramae Hospital (SNUBH). All living donor LTs (LDLTs) and deceased donor LTs (DDLTs) were included. Of the 2,201 patients, 743 were excluded for being $<18$ years of age at surgery ( $\mathrm{n}=189)$, dying within 1 month of LT $(\mathrm{n}=21)$, requiring re-transplantation not associated with HBV infection $(\mathrm{n}=14)$, or having incomplete clinical data including loss to follow-up for analysis $(\mathrm{n}=519)$. Therefore, 1,458 patients were eligible for enrollment. Information of the deceased donors was obtained from their medical records from the Korean Network for Organ Sharing. Serologic tests for HBV antigen/antibody status were conducted for all living or deceased donors. For the recipients, age, sex, etiology of liver disease, Model for End-Stage Liver Disease (MELD) score, antibody status, and transplantation type (DDLT or LDLT) were included as variables. For the donors, age, sex, and $\mathrm{HBsAb}$ positivity were included as variables. The study was conducted in accordance with the Declaration of Helsinki (as revised in 2013). This study was conducted at two hospitals and was approved by the Institutional Review Boards of SNUH (H2008-193-1154) and SNUBH (20-2021-17). The need for informed consent was waived by the review boards due to the retrospective nature of the study.

De novo $\mathrm{HBV}$ infection was defined as the detection of serum HBsAg, with or without HBV DNA detection in a recipient who was HBsAg-negative before transplantation. The liver donors were divided into two groups based on the presence of core-antibody, and the HBsAg-negative recipients were categorized into four groups based on their $\mathrm{HBcAb}$ and HBsAb status. The number of allocations of core-positive livers to each of the four recipient groups was determined (Figure 1). The liver biopsy was performed within 1month before and after the detection of de novo $\mathrm{HBV}$ infection. The risk factors for the development of de novo $\mathrm{HBV}$ infection in recipients with core-antibody 


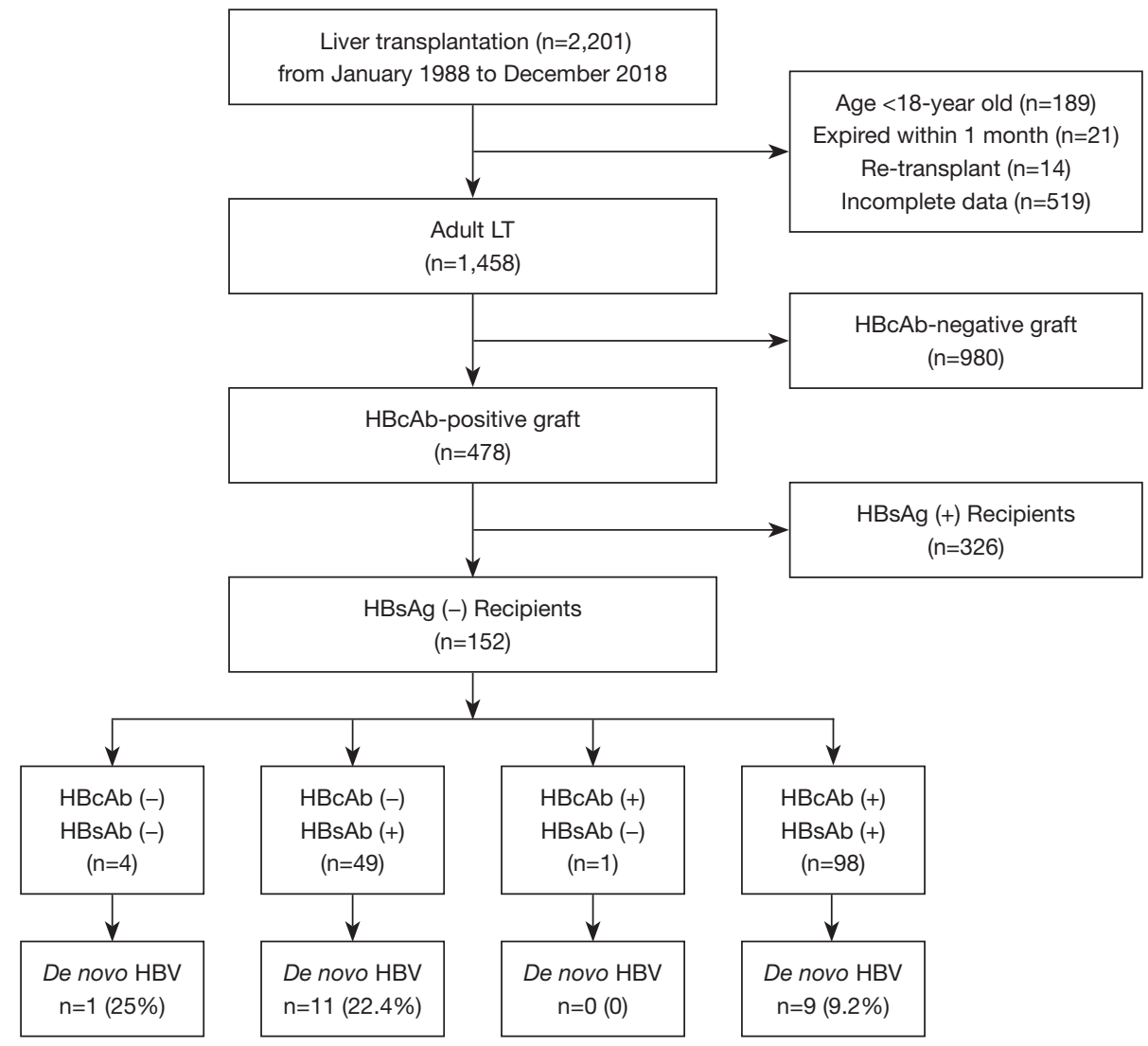

Figure 1 Flow chart demonstrating the study population. LT, liver transplantation; HBcAb, hepatitis B core antibody; HBsAg, hepatitis B surface antigen.

positive grafts were analyzed.

\section{Immunosuppression regimen}

For the induction, $40 \mathrm{mg}$ basiliximab (Simulect, Novartis, Montreal, QC, Canada) was administered on the day of surgery and postoperative day 4 . The maintenance regimen for immunosuppression included tacrolimus, mycophenolate mofetil (500 $\mathrm{mg}$ twice a day), and corticosteroids (methylprednisolone for the immediate posttransplant period and then prednisolone). The target serum concentration of tacrolimus was $8-12 \mathrm{ng} / \mathrm{mL}$ for the first 6 months post-transplant and $6-8 \mathrm{ng} / \mathrm{mL}$ for the following 6 months.

\section{De novo $H B V$ prophylaxis protocol}

Prophylaxis for de novo HBV infection at both centers was conducted according to the same protocols. In HBsAg- negative recipients, if either the donor or recipient was core-antibody positive, 4,000 IU HBIG (Hepabig, Green Cross, Yongin, South Korea) was intravenously administered during the anhepatic phase in the operating room and daily until postoperative day 3 (Figure S1). This protocol was followed in all patients. Subsequently, 4,000 IU HBIG was injected to maintain a trough serum HBsAb titer of $\geq 100 \mathrm{IU} / \mathrm{L}$ at the outpatient clinic. HBV vaccine (Euvax, LG Bioscience, Seoul, South Korea) was administered to only 14 patients, with a target maintenance HBsAb titer of approximately $>100 \mathrm{IU} / \mathrm{L}$ at the time of tapering of the steroid after LT, according to a practitioner's decision at the outpatient clinic.

\section{Follow-up}

Regular follow-up after LT was performed every 1-2 weeks for the first month, then once a month until 2-4 months, and then every 3-4 months. Routine laboratory tests 
included serum HBsAg and HBsAb titers. The HBV DNA viral load was determined at the time when a positive HBsAg result was obtained following LT.

\section{Statistical analyses}

Statistical analyses were performed using SPSS version 27.0 for Windows (IBM Corporation, Armonk, NY, USA). Continuous variables were compared using Student's $t$-test. Categorical variables were analyzed using the chi-square or Fisher's exact tests. Multivariate analysis was performed with logistic regression for analyzing risk factors of de novo HBV infection development. The Kaplan-Meier method was used to determine the overall survival and time to detection of de novo HBV, and the survival curves were compared using the log-rank test. A $\mathrm{P}$ value $<0.05$ was considered to be statistically significant.

\section{Results}

\section{Demographics of HBsAg-negative recipients}

A total of 1,458 LTs in adult recipients were analyzed. Among 526 HBsAg-negative recipients (36.1\%), 152 (28.9\%) patients received $\mathrm{HBcAb}$-positive grafts. Within this $\mathrm{HBcAb}-$ positive group, the mean age was 52.8 years, and 81 (53.3\%) were male. Alcoholic liver disease and $\mathrm{HCV}$ hepatitis were observed in $47(30.9 \%)$ and $44(28.9 \%)$ recipients, respectively. The mean MELD score was 20.1. The proportion of core-positive grafts was greater in the DDLT pool than in the LDLT pool $(45.2 \%$ vs. $24.9 \%, \mathrm{P}<0.001)$. De novo $\mathrm{HBV}$ infection was significantly higher in recipients with a core-positive graft $(13.8 \%$ vs. $1.3 \%, \mathrm{P}<0.001)$. The mean age of donors with core positivity was significantly higher than that of core-negative donors (44.3 vs. 34.1 years, $\mathrm{P}<0.001)$. Detailed data are presented in Table 1.

\section{De novo $\mathrm{HBV}$ infection rate by recipient antibody status}

Of the 1,458 transplants, 478 (32.8\%) used HBcAb-positive grafts, and HBsAg-positive and -negative recipients were allocated $326(68.2 \%)$ and 152 (31.8\%) HBcAb-positive grafts, respectively. According to the recipient antibody status, the de novo infection rate was different. De novo $\mathrm{HBV}$ was diagnosed in 1/4 (25\%) of HBcAb- and HBsAbnegative recipients, 11/49 (22.4\%) of HBcAb-negative and $\mathrm{HBsAb}$-positive recipients, $0 / 1$ of $\mathrm{HBcAb}$-positive and HBsAb-negative recipients, and 9/98 (9.2\%) of HBcAb- and HBsAb-positive recipients (Figure 1).

\section{Risk factors for de novo HBV infection}

The risk factors associated with de novo HBV infection are reported in Table 2. In the univariate analysis, $\mathrm{HBcAb}-$ negative recipients were more likely to develop de novo HBV infection than $\mathrm{HBcAb}$-positive recipients $(22.6 \%$ vs. $9.1 \%$, $\mathrm{P}=0.021)$. The incidence of de novo HBV infection did not differ based on the recipient's HBsAb status $(\mathrm{P}=0.530)$. A higher MELD score was significantly associated with de novo $\mathrm{HBV}$ infection (23.8 vs. 19.5, $\mathrm{P}=0.047$ ); however, it was not statistically significant in multivariate analysis. Age, sex, or etiology of liver disease of the recipients; type of transplantation; or HBsAb positivity of donors had no effect on the de novo $\mathrm{HBV}$ infection.

\section{Patients with de novo HBV infection}

Analysis of patients who developed de novo HBV infection is described in Table 3. The median follow-up duration for enrolled patients was 69 months (range, 29-165 months). The mean time for the detection of serum HBsAb positivity was 18 months (range, 8-55 months). Of the 21 patients, 2 $(9.5 \%)$ did not undergo any treatment due to the immediate seroconversion at a sequential laboratory test or surgeon's discretion. Among the patients who were treated, 12 (63.2\%) were treated using NA monotherapy and 7 (36.8\%) patients were treated with a combination of NA and HBIG. The median treatment duration was 41 months (range, $0-105$ months). Seroconversion was achieved in seven patients. No patient died of de novo HBV infection.

\section{Hepatitis B surface antibody (anti-HBs) titer at diagnosis of de novo HBV infection}

The median HBsAb titer of patients who developed de novo infection at transplant and the diagnosis was $46.0 \mathrm{IU} / \mathrm{L}$ (range, 2.0-1,000.0 IU/L) and 65.0 IU/L (range, 0-960.8 IU/L), respectively (Table 3). Figure 2 shows the time to detection for serum HBsAg positivity and HBsAb titers at the time of detection. Two patients showed an HBsAb titer of $>100.0$ IU/L; however, 19 patients had an HBsAb titer of $\leq 100.0$ IU/L at the time of detection.

\section{Posttransplant $H B V$ vaccination and response}

Of the 152 recipients who received $\mathrm{HBcAb}$-positive grafts, 
Table 1 Baseline characteristics of HBsAg-negative recipients

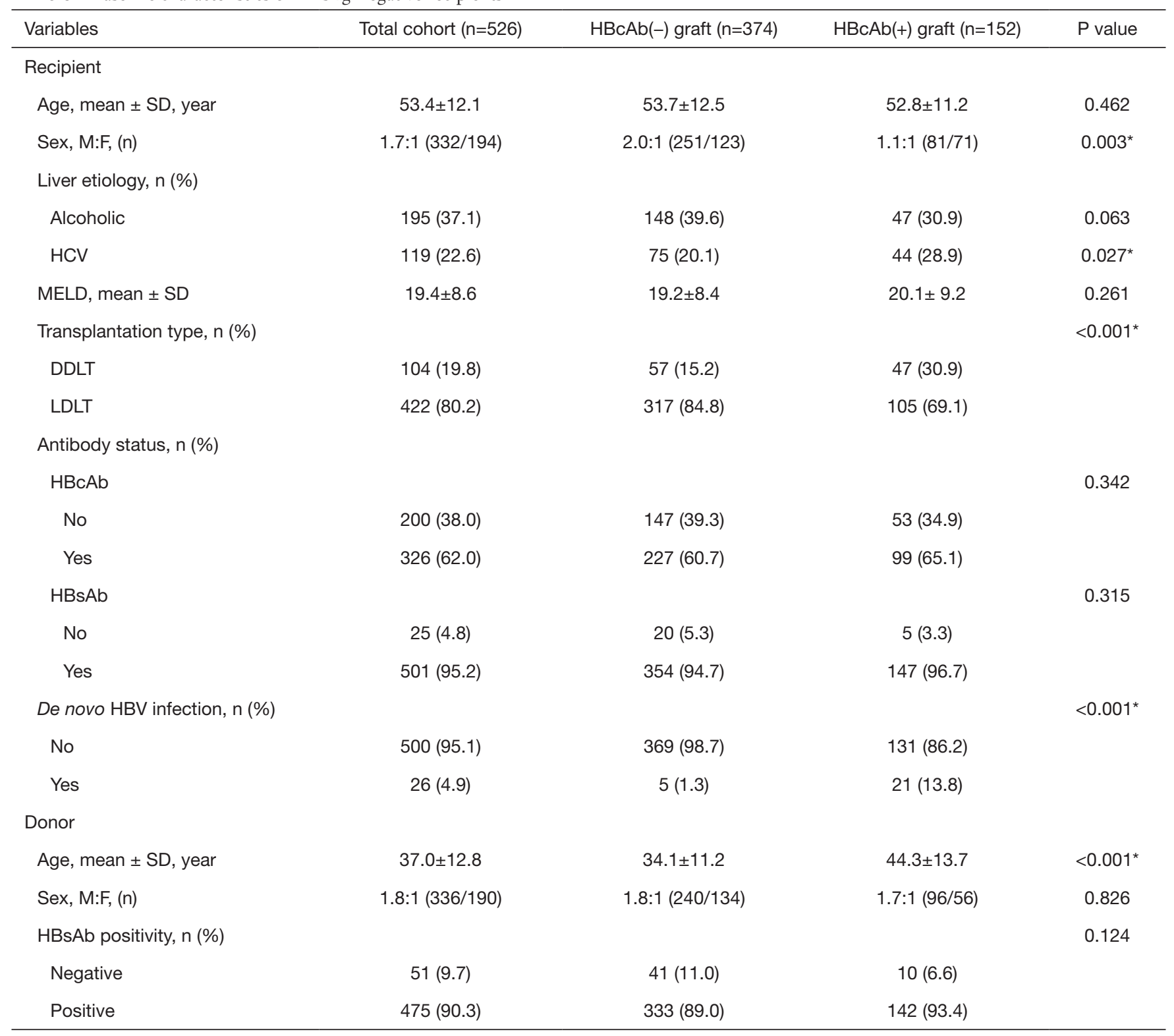

*, P value considered statistically significant $(<0.05)$. HBsAg, hepatitis B surface antigen; HBcAb, hepatitis B core antibody; HCV, hepatitis C virus; MELD, Model for End-Stage Liver Disease; DDLT, deceased donor liver transplantation; LDLT, living donor liver transplantations; $\mathrm{HBV}$, hepatitis B virus;

$14(9.2 \%)$ were vaccinated after LT with a median of 2 doses (range, 1-10 doses) according to the surgeon's preference. The median HBsAb titer before vaccination was 39.2 IU/L. Of the 14 vaccinated patients, 8 had a response, and their HBsAb titers were maintained above 100.0 IU/L from the time of vaccination. HBsAb $<100.0 \mathrm{IU} / \mathrm{L}$ was observed in 6 of the 14 patients, and de novo HBV infection developed in 4 of them.

\section{Discussion}

The strategies for the prevention of de novo HBV infection after LT varies in the current clinical practice $(8,15-17)$. Previous studies have shown varying risks of de novo HBV infection with incidences ranging from 0 to $25 \%$ with poor survival $(7,18)$. However, most of these studies were conducted in Western countries where the prevalence of 
Table 2 Risk factors for de novo HBV in HBsAg-negative recipients with core-positive grafts ( $\mathrm{n}=152)$

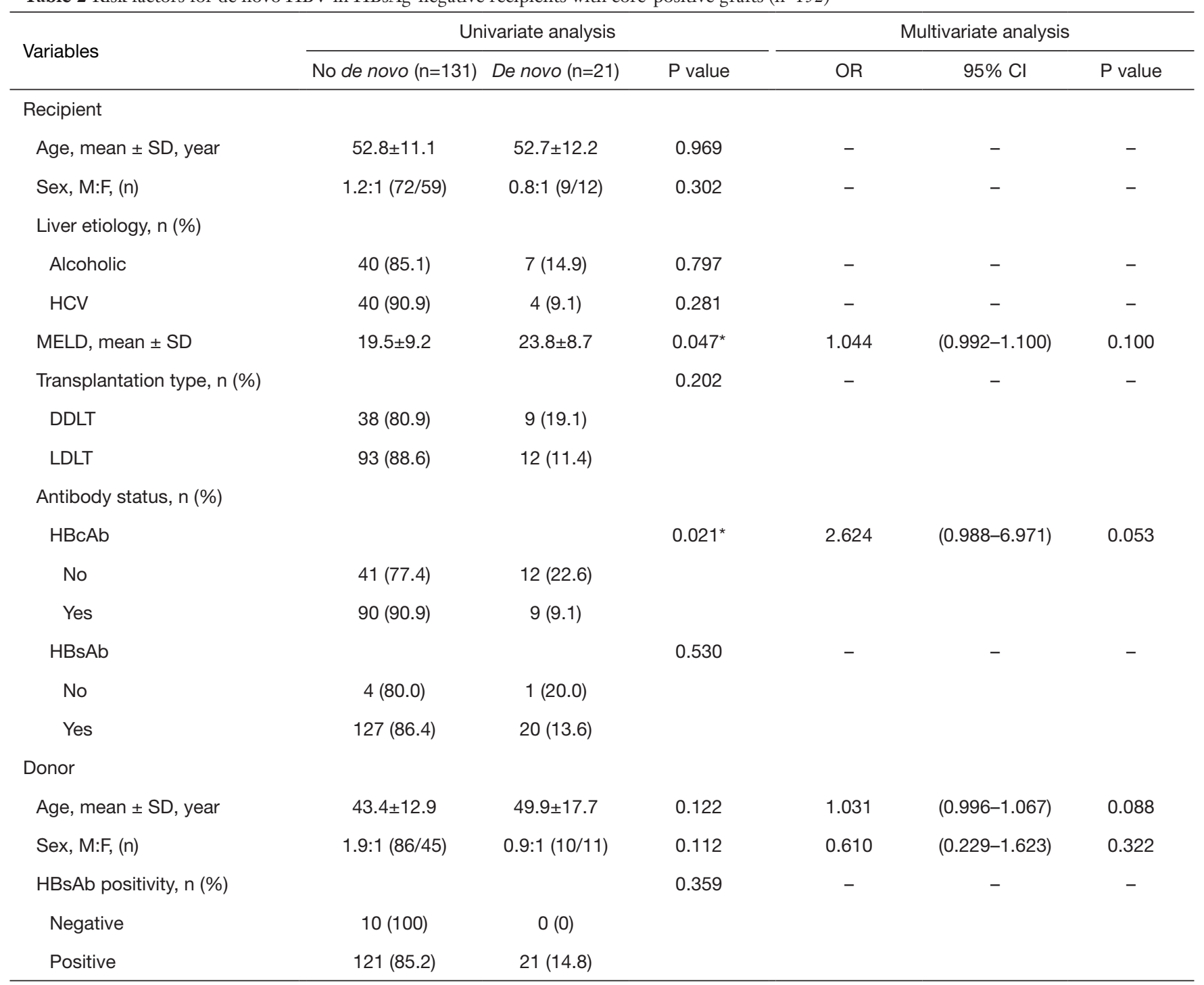

*, P value considered statistically significant (<0.05). HBV, hepatitis B virus; HBsAg, hepatitis B surface antigen; HCV, hepatitis C virus; MELD, Model for End-Stage Liver Disease; DDLT, deceased donor liver transplantation; LDLT, living donor liver transplantations.

HBV infection is low and core-positive grafts are regarded as extended grafts.

The incidence of de novo HBV has been decreasing because of the empirical use of NA and HBIG in Western countries in recent years (19-21). Cholongitas et al. revealed that lamivudine monotherapy $(2.6 \%)$ or HBIG and the lamivudine combination regimen (2.8\%) markedly decreased the de novo infection rates compared with HBIG monoprophylaxis (19\%) in HBsAg-negative recipients (6). Despite the efficacy and convenience to administer, the cost issues and side-effects resulting from the life-long NA prophylaxis regimen remain controversial.

This large cohort study elucidated long-term outcomes of HBIG monotherapy preventing de novo HBV infection after LT using $\mathrm{HBcAb}$-positive liver grafts in an $\mathrm{HBV}$ endemic area. $\mathrm{HBcAb}$-negative recipients were more likely to develop de novo HBV infection than HBcAbpositive recipients. A higher MELD score was significantly associated with de novo HBV infection.

According to our data, the incidence of de novo HBV infection was $13.8 \%$ in HBsAg-negative recipients of corepositive grafts with HBIG monoprophylaxis. Some studies 
Table 3 Analysis of patients with de novo HBV infection

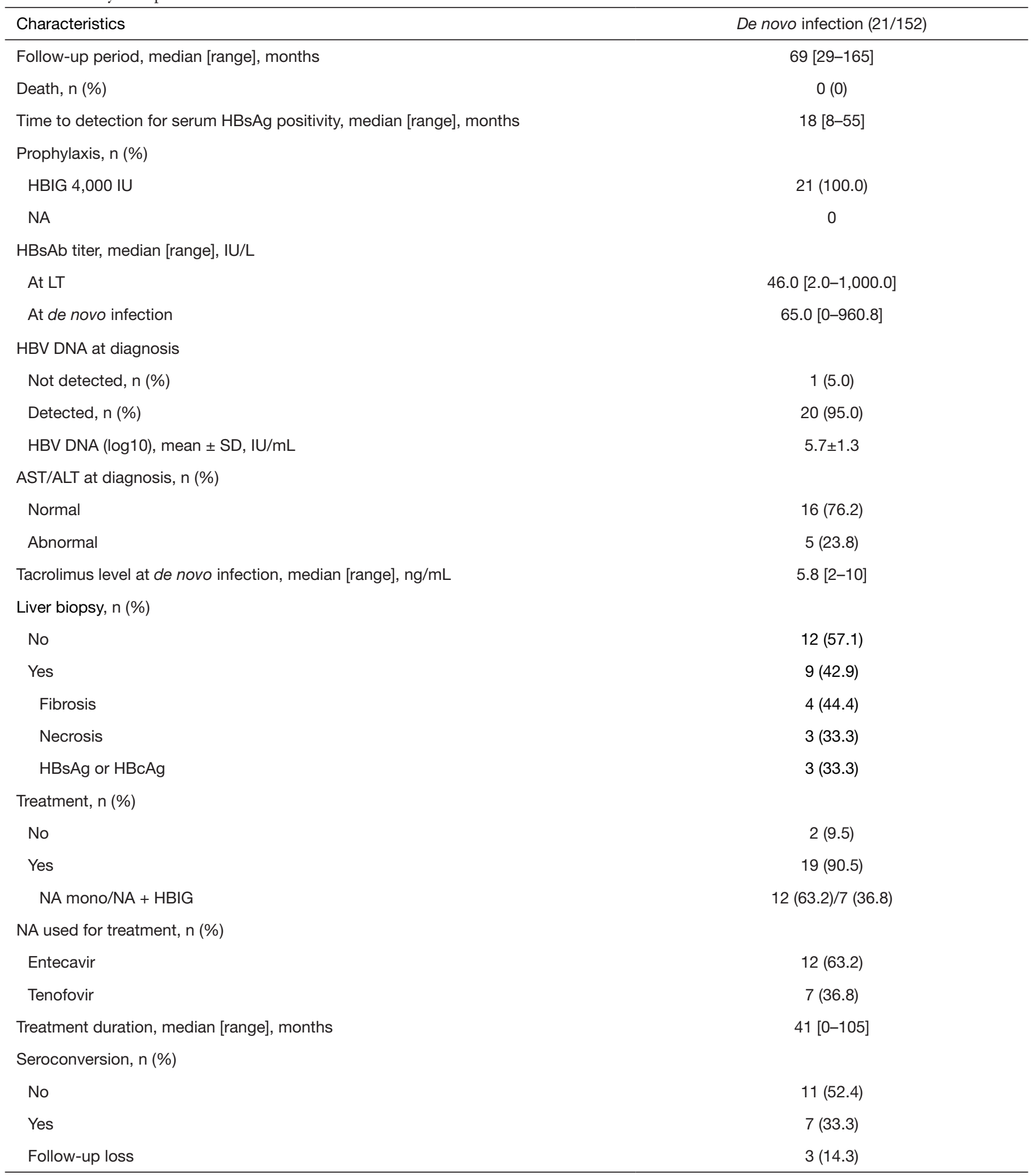

AST >50 or ALT >50 means abnormal AST or ALT. HBV, hepatitis B virus; HBsAg, hepatitis B surface antigen; LT, liver transplantation; AST, aspartate aminotransferase; ALT, alanine aminotransferase; NA, nucleoside analogs; HBIG, hepatitis B immunoglobulin. 


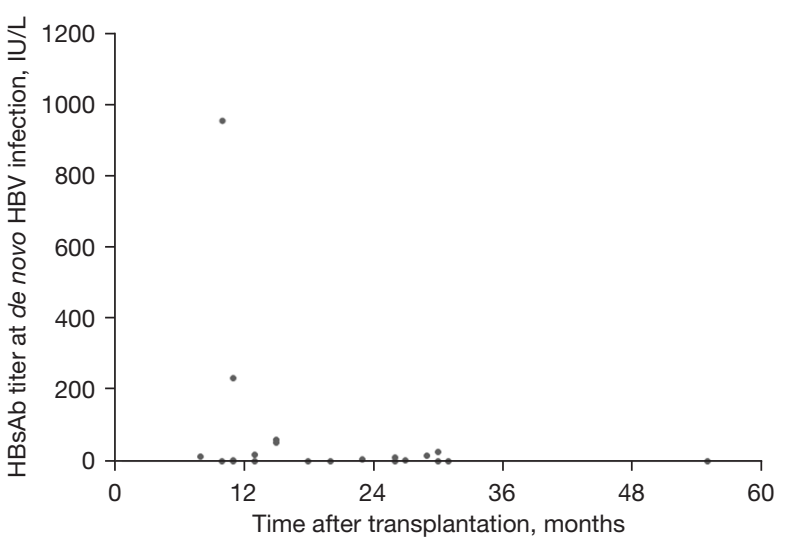

Figure 2 Anti-HBs titer at diagnosis of de novo $\mathrm{HBV}$ infection. Anti-HB, hepatitis B surface antibody; HBV, hepatitis B virus.

performed in South Korea reported de novo HBV infection rates from $7.7 \%$ to $12.5 \%$ without any prophylaxis (22). These infection rates are considered high in the era of NA. However, we revealed that the de novo rate varies according to the recipient's antibody status from $0 \%$ to $25 \%$. Although the de novo infection rate with HBIG monoprophylaxis was higher than that with NA, administration of NA for all $\mathrm{HBsAg}$ recipients with $\mathrm{HBcAb}$-positive grafts may be an overtreatment, given that the Eastern countries are $\mathrm{HBV}$ prevalent.

The medical insurance system in South Korea covers monthly infusion of HBIG in recipients of core-positive graft. By contrast, in Western countries HBIG is expensive and not covered with medical insurance. This may explain the long-term practice that has used HBIG monotherapy during perioperative and postoperative periods over decades. The prophylactic use of NA after LT performed in HBsAg-negative recipients does not have full insurance coverage. This has resulted in NA therapy being initiated in patients following the diagnosis of de novo $\mathrm{HBV}$ infection. Given these cost issues, HBIG for prophylaxis may be a proper strategy in terms of cost and effectiveness.

In this study, not all patients with de novo HBV presented abnormal aspartate transaminase and alanine transaminase levels. Thus, subclinical de novo HBV infection may arise because of the state of immune equilibrium, without any liver damage. This may pose a challenge with respect to the optimal timing of prevention using NA for de novo HBV. Additionally, liver graft survival was $100 \%$, and no definitive liver damage was observed in some biopsy cases in this study. Therefore, NA therapy may be withheld despite detecting serum HBsAg positivity.
$\mathrm{HBV}$-naive patients, with both $\mathrm{HBcAb}$ and $\mathrm{HBsAb}$ negativity, were the most vulnerable for de novo infection in this study, which is in accordance with previous results $(6,22)$. One of four (25\%) patients developed de novo infection with the HBIG-only protocol. Previous results and this study suggest that the HBV-naive recipients may need to be identified as a high-risk group of de novo HBV infection (16). In addition to the subgrouping, conduction of a stronger protocol for de novo $\mathrm{HBV}$ prophylaxis, such as a higher dose of HBIG or combination with NA, may be required.

This study also demonstrated that vaccinated recipients without a history of $\mathrm{HBV}$ infection, who are core-negative and surface antibody-positive before transplantation, have the second highest risk for de novo $\mathrm{HBV}$ infection. Eleven of $49(22.4 \%)$ core-negative, surface antibody-positive recipients developed de novo infection retrospectively. $\mathrm{HBsAb}$-positive recipients had a lower risk of de novo infection, although the difference was not statistically significant ( $13.6 \%$ vs. $20.0 \%, \mathrm{P}=0.530)$. Owing to a high prevalence of anti-HBs positivity in South Korea, the incidence of de novo infection is similar to that in HBVnaive patients, even in preoperatively vaccinated recipients. Therefore, the presence of anti-HBs before transplant cannot ensure an absolute immune barrier to de novo $\mathrm{HBV}$ infection.

In addition to the ineffectiveness of preoperative vaccination, posttransplant vaccination did not demonstrate an outstanding record against de novo infection. Wang et al. reported that postoperative $\mathrm{HBV}$ vaccination was only effective against de novo $\mathrm{HBV}$ infection when preoperative anti-HBs was $>1,000.0 \mathrm{IU} / \mathrm{L}$; however, lamivudine may be continued if the postoperative HBsAb titer is $<100.0 \mathrm{IU} / \mathrm{L}$, even if a postoperative $\mathrm{HBV}$ vaccine was administered to those recipients (17). Fourteen patients were vaccinated during postoperative periods, especially during tapering of steroids, in our data. However, 6 of 14 did not have any benefit for developing HBsAb titer from posttransplant vaccination. Cholongitas et al. also revealed $\mathrm{HBV}$ vaccination after LT to be an ineffective strategy as the de novo $\mathrm{HBV}$ infection rate was $100 \%$ with $\mathrm{HBV}$ vaccination monoprophylaxis (6).

The prevalence of core-antibody-positive organs was significantly greater in DDLTs than in LDLTs in our study. Limitation in choice with regard to grafts in urgent situations of DDLTs may bring about this result. Registered deceased liver donors are screened for HBV-related serological examinations in practice. We also recommend 
that transplant centers encourage the systemic sharing of information about donor HBV-related serological results and maintain the surveillance for de novo HBV infection after transplant.

We found that a higher MELD score of recipients was significantly related to core-positivity of grafts and the development of de novo infection. A previous study similarly reported a Child-Pugh score of LT had a significant effect on the occurrence of de novo infection (18). Patients with greater morbidity at transplantation may be prone to de novo $\mathrm{HBV}$ infection due to their immunocompromised status.

The time to detection of de novo HBV was diverse, ranging from 8 to 55 months. As the occurrence of $d e$ novo infection is sporadic, regular follow-up of serum hepatitis B viral markers such as HBsAg or HBsAb are needed, especially in high-risk patients at least for several posttransplant years.

This study, to the best our knowledge, is the largest single-center cohort study to evaluate the risk and outcomes of de novo HBV infection based on predetermined HBIG monoprophylaxis protocol. This study was conducted in an $\mathrm{HBV}$ endemic area and in clinical practice with increased use of core-positive grafts. A long follow-up period was one of the strengths of the current study.

However, this study has some limitations. This study was conducted at a single institution and the number of de novo HBV patients after LT is still low. We did not routinely assess the serum HBsAg for surveillance, which resulted in a significant loss of data for analysis. Patients with a maintenance HBsAb titer of $\geq 200.0 \mathrm{IU} / \mathrm{mL}$ were regarded to be without infection. The median followup period for the patients with de novo infection was not sufficient to evaluate the long-term result of de novo HBV infection in the post-transplant population. This study was performed at a tertiary center which has maintained the HBIG monoprophylaxis protocol for decades, which resulted in the lack of comparison between the NA and HBIG approaches for the de novo HBV prevention. This also affects the interpretation of HBIG benefits.

In the era of NA, HBIG-only prophylaxis may not be sufficient to prevent de novo $\mathrm{HBV}$ development in not only $\mathrm{HBV}$-naive patients but also recipients who had been vaccinated without a history of $\mathrm{HBV}$ infection. However, de novo $\mathrm{HBV}$ infection did not affect patient survival. With surveillance for de novo $\mathrm{HBV}$ infection, close monitoring of viral serum markers, and appropriate NA initiation, de novo $\mathrm{HBV}$ infection can be prevented with HBIG protocol and treated with NA administration from the time of diagnosis appropriately. Further investigation concerning the detailed regimen or timeline for monitoring will help establish a tailored strategy for de novo $H B V$ prevention.

\section{Acknowledgments}

Funding: None.

\section{Footnote}

Reporting Checklist: The authors have completed the STROBE reporting checklist. Available at https://atm. amegroups.com/article/view/10.21037/atm-21-4311/rc

Data Sharing Statement: Available at https://atm.amegroups. com/article/view/10.21037/atm-21-4311/dss

Conflicts of Interest: All authors have completed the ICMJE uniform disclosure form (available at https://atm. amegroups.com/article/view/10.21037/atm-21-4311/coif). The authors have no conflicts of interest to declare.

Ethics Statement: The authors are accountable for all aspects of the work in ensuring that questions related to the accuracy or integrity of any part of the work are appropriately investigated and resolved. The study was conducted in accordance with the Declaration of Helsinki (as revised in 2013). The study was approved by the Institutional Review Board of Seoul National University Hospital (H-2008-193-1154) and Seoul National University Boramae Hospital (20-2021-17). The need for informed consent was waived by the review boards due to the retrospective nature of the study.

Open Access Statement: This is an Open Access article distributed in accordance with the Creative Commons Attribution-NonCommercial-NoDerivs 4.0 International License (CC BY-NC-ND 4.0), which permits the noncommercial replication and distribution of the article with the strict proviso that no changes or edits are made and the original work is properly cited (including links to both the formal publication through the relevant DOI and the license). See: https://creativecommons.org/licenses/by-nc-nd/4.0/.

\section{References}

1. Hwang S, Moon DB, Lee SG, et al. Safety of anti-hepatitis $\mathrm{B}$ core antibody-positive donors for living-donor liver 
transplantation. Transplantation 2003;75:S45-8.

2. Perrillo R. Hepatitis $B$ virus prevention strategies for antibody to hepatitis B core antigen-positive liver donation: a survey of North American, European, and Asian-Pacific transplant programs. Liver Transpl 2009; 15:223-32.

3. Roche B, Samuel D, Gigou M, et al. De novo and apparent de novo hepatitis B virus infection after liver transplantation. J Hepatol 1997;26:517-26.

4. Brandl A, Stolzlechner P, Eschertzhuber S, et al. Inferior graft survival of hepatitis B core positive grafts is not influenced by post-transplant hepatitis B infection in liver recipients--5-year single-center experience. Transpl Int 2016;29:471-82.

5. Brock GN, Mostajabi F, Ferguson N, et al. Prophylaxis against de novo hepatitis $\mathrm{B}$ for liver transplantation utilizing hep B core (+) donors: does hepatitis B immunoglobulin provide a survival advantage? Transpl Int 2011;24:570-81.

6. Cholongitas E, Papatheodoridis GV, Burroughs AK. Liver grafts from anti-hepatitis B core positive donors: a systematic review. J Hepatol 2010;52:272-9.

7. Angelico M, Nardi A, Marianelli T, et al. Hepatitis B-core antibody positive donors in liver transplantation and their impact on graft survival: evidence from the Liver Match cohort study. J Hepatol 2013;58:715-23.

8. Wong TC, Fung JY, Cui TY, et al. Liver transplantation using hepatitis B core positive grafts with antiviral monotherapy prophylaxis. J Hepatol 2019;70:1114-22.

9. Braithwaite AM, Garcia M, Rubin A, et al. Prophylaxis of De Novo Hepatitis B Virus Infection With Lamivudine in HBsAg-Negative Naive Recipients of Hepatitis B Core Antibody-Positive Livers: A 13-Year Single-Center Experience. Liver Transplantation 2014;20:S312.

10. Dodson SF, Bonham CA, Geller DA, et al. Prevention of de novo hepatitis $B$ infection in recipients of hepatic allografts from anti-HBc positive donors. Transplantation 1999;68:1058-61.

11. Han JH, Kim DG, Na GH, et al. De novo hepatitis B virus infection developing after liver transplantation using a graft positive for hepatitis B core antibody. Ann Surg Treat Res 2015;89:145-50.

12. Lee KW, Lee DS, Lee HH, et al. Prevention of de novo hepatitis B infection from HbcAb-positive donors in living donor liver transplantation. Transplant Proc 2004;36:2311-2.

13. Takemura N, Sugawara Y, Tamura S, et al. Liver transplantation using hepatitis B core antibody-positive grafts: review and university of Tokyo experience. Dig Dis Sci 2007;52:2472-7.

14. Terrault NA, Lok ASF, McMahon BJ, et al. Update on Prevention, Diagnosis, and Treatment of Chronic Hepatitis B: AASLD 2018 Hepatitis B Guidance. Clin Liver Dis (Hoboken) 2018;12:33-4.

15. Neff GW, O'brien CB, Nery J, et al. Outcomes in liver transplant recipients with hepatitis $B$ virus: resistance and recurrence patterns from a large transplant center over the last decade. Liver Transpl 2004;10:1372-8.

16. Skagen CL, Jou JH, Said A. Risk of de novo hepatitis in liver recipients from hepatitis-B core antibody-positive grafts - a systematic analysis. Clin Transplant 2011;25:E243-9.

17. Wang SH, Loh PY, Lin TL, et al. Active immunization for prevention of De novo hepatitis B virus infection after adult living donor liver transplantation with a hepatitis B core antigen-positive graft. Liver Transpl 2017;23:1266-72.

18. Prieto M, Gómez MD, Berenguer M, et al. De novo hepatitis B after liver transplantation from hepatitis B core antibody-positive donors in an area with high prevalence of anti-HBc positivity in the donor population. Liver Transpl 2001;7:51-8.

19. Nery JR, Nery-Avila C, Reddy KR, et al. Use of liver grafts from donors positive for antihepatitis B-core antibody (anti-HBc) in the era of prophylaxis with hepatitis-B immunoglobulin and lamivudine. Transplantation 2003;75:1179-86.

20. Ben-Ari Z, Mor E, Tur-Kaspa R. Experience with lamivudine therapy for hepatitis $\mathrm{B}$ virus infection before and after liver transplantation, and review of the literature. J Intern Med 2003;253:544-52.

21. Chotiyaputta W, Pelletier SJ, Fontana RJ, et al. Longterm efficacy of nucleoside monotherapy in preventing $\mathrm{HBV}$ infection in HBsAg-negative recipients of anti-HBcpositive donor livers. Hepatol Int 2010;4:707-15.

22. Kim HY, Choi JY, Park CH, et al. Adult Living Donor Liver Transplantation Using Hepatitis B Core AntibodyPositive Grafts in Korea, a Hepatitis B-endemic Region. Gut Liver 2011;5:363-6.

Cite this article as: Jung HS, Choi Y, Yoon KC, Hong SY, Suh S, Hong K, Han ES, Lee JM, Hong SK, Yi NJ, Lee KW, Suh KS. Hepatitis B immunoglobulin prophylaxis for de novo hepatitis B infection in liver transplantation: a 30-year experience. Ann Transl Med 2022;10(5):243. doi: 10.21037/atm$21-4311$ 


\section{Supplementary}

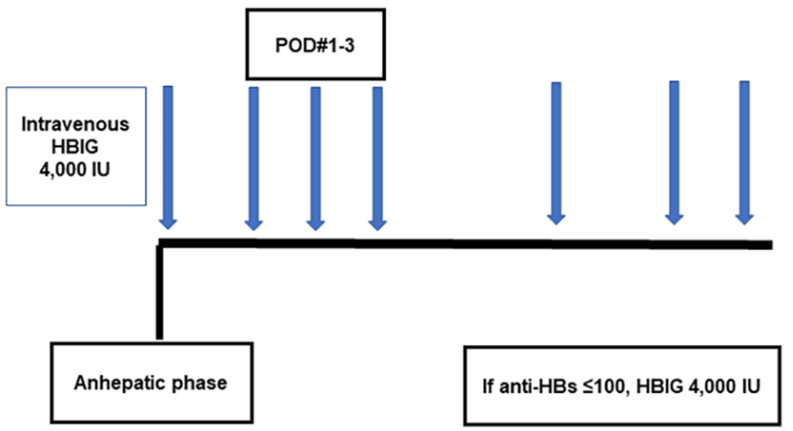

Figure S1 De novo HBV prophylaxis protocol in SNUH. HBV, hepatitis B virus; SNUH, Seoul National University Hospital. 